\title{
Clinical and Radiological Mid-to-Long-term Outcomes Following Ankle Arthrolysis
}

\author{
FREYA-ISABELLE ROHLFING ${ }^{1,2}$, ULRICH WIEBKING ${ }^{1,2}$, \\ PADHRAIG F. O'LOUGHLIN ${ }^{3}$, CHRISTIAN KRETTEK ${ }^{2}$ and RALPH GAULKE ${ }^{1,2}$ \\ ${ }^{1}$ Section of Upper Extremity, Foot and Rheuma Surgery, Trauma Department, \\ Medical School Hannover (MHH), Hannover, Germany; \\ ${ }^{2}$ Trauma Department, Medical School Hannover (MHH), Hannover, Germany; \\ ${ }^{3}$ Mater Hospital, Cork, Ireland
}

\begin{abstract}
Background: The aim of this study was to evaluate the clinical and radiographic success of arthrolysis surgery and the risk of progression of osteoarthrosis at the ankle joint. Materials and Methods: In a retrospective clinical and radiological study, with a minimum follow-up of 24 months, the pain level and quality of living were evaluated. Results: Following arthrolysis of the ankle joint, $16 \%$ of patients required ankle fusion within 2 years. Women had a higher quality-of-life in terms of Foot Function Index. Younger patients scored higher in both quality-of-life and function scores. Radiographic osteoarthrotic changes and the specific follow-up interval did not correlate with clinical outcome. Conclusion: Fewer than 20\% of patients required ankle fusion. Female gender and young age had a positive impact. Preoperative radiography and the postsurgical interval are poorly predictive for the progression of osteoarthrosis.
\end{abstract}

Osteoarthrosis of the ankle joint can have a significant, detrimental impact on quality-of-life as it typically limits mobility and causes pain. It may be caused by trauma, autoinflammatory conditions such as rheumatoid arthritis, overload and instability. Genetic factors for early-onset polyarthrosis have been described $(1,2)$.

Evidence-based literature (grade B) supports ankle arthroscopy for the treatment of ankle impingement, osteo-

This article is freely accessible online.

Correspondence to: Prof. Dr. med. Ralph Gaulke, Leiter der Sektion Obere Extremität, Fuß- und Rheumachirurgie, Unfallchirurgische Klinik, Medizinische Hochschule Hannover, Carl-Neuberg-Str. 1, D-30625 Hannover, Germany. E-mail: gaulke.ralph@mhhannover.de

Key Words: Ankle osteoarthritis, ankle arthrolysis, long-term follow-up, tibiotalar impingement. chondral lesions and mild osteoarthrosis (3). Symptomatic early-onset ankle joint osteoarthrosis with limitation of ROM is a common indication for open or arthroscopic arthrolysis. This procedure involves synovectomy, resection of intraarticular scar tissue, free bodies and osteophytes (mostly located on the anterior talar and tibial areas), chondroplasty with/without arthroplasty. In recent decades, an improvement in surgical instruments and surgeons' experience has led to an evolution of the gold standard from open to arthroscopic ankle joint release. Few studies (4-7) have analyzed outcomes at long- and short-term follow-up, but most of these recorded good results. Post-operative complications related to ankle arthroscopy have been described in the literature and include most commonly cutaneous nerve injury and superficial postoperative infection without significant long-term sequelae from either $(8,9)$.

Our aim was to study postoperative changes in function and pain following arthrolysis surgery of the ankle joint. The study endpoint was defined as revision surgery at the ankle joint, such as repeat arthrolysis, ankle arthroplasty, or arthrodesis. Special focus was placed on the correlation between pain, and radiological and clinical findings.

\section{Patients and Methods}

This retrospective clinical and radiological single-centre study was performed with approval of the local Ethics Committee (No. 6927). All patients who underwent open or arthroscopic ankle arthrolysis at the Trauma Department of Medical School Hannover between 2000 and 2014 were included. The minimum follow-up after surgery was 2 years. Senior orthopaedic foot and ankle surgeons performed arthroscopic surgery with a shaver and a $6 \mathrm{~mm}$ Lambotte gouge with visualisation enabled by a $4 \mathrm{~mm} 30^{\circ}$ oblique arthroscope via anterolateral and anteromedial portals $(10,11)$. Open surgery was performed via an anterolateral approach.

Patients with septic arthritis, neoplastic conditions, age less than 18 years, open physes or previous ankle surgery were excluded.

Patients who underwent ankle fusion (primary endpoint) before participating in this study were asked to answer the questionnaires 
whilst recalling their symptoms at 2 weeks before ankle arthrodesis. These patients were not invited for clinical and radiological examination. The results of the questionnaires were factored in with regard to the survival of the joint after arthrolysis.

Standardised questionnaires for function and quality-of-life evaluation were employed: Foot Function Index: FFI-pain: 0 points (best result) to 72 points (worst result), FFI-function: 0 points (best result) to 90 points (worst result) (11); The American Orthopedic Foot and Ankle Score (AOFAS): which covers three categories: pain (40 points), function (50 points) and alignment (10 points), 100 points means excellent outcomes and 0 points worst outcome (12); and Hannover score (20 points for the best, 100 for the worst outcome) (13).

The patients were asked about their level of pain at three time points: pre-surgery, 1 month after surgery and at current follow-up, via visual analogue scale [VAS; scale 0 (no pain) to 10 (worst pain)]. In order to evaluate the progression-free-interval after surgery, patients were asked to state retrospectively how long the post-operative VAS remained stable.

Clinical and radiological examinations were performed by FIR under the supervision of RG and UW. Radiographs of the ankle in mortise view and weightbearing lateral views of the foot were performed. In order to identify the progression of osteoarthrosis in the ankle joint and the adjacent joints after surgery, the radiographs at follow-up were compared to those taken postoperatively.

The grade of osteoarthrosis in the ankle, subtalar, and Chopart joints was evaluated using the Kellgren-Lawrence score (KLS) (14, 15). An increase of the KLS score between the time of surgery and the most recent follow-up was graded as progression of osteoarthrosis.

A mirror-table was used for semi-quantitative measuring of the weight-bearing area of the plantar aspect of the foot. The mirrortable footprint and the hindfoot axis (measured with a goniometer) were documented photographically.

The range of motion (ROM) of the hip, knee, ankle, subtalar and Chopart joints was measured with a goniometer, on both sides, to detect differences between the treated and untreated foot. Hypertrophy or atrophy of muscle groups and soft-tissue swelling were detected by differences in limb diameter at 10 and $20 \mathrm{~cm}$ above and $15 \mathrm{~cm}$ below the medial joint line of the knee respectively, at the slimmest part of the lower leg, at the ankle and at the forefoot. The power of the tibialis anterior and posterior, peroneus longus, triceps surae, and the extensor and flexor hallucis and digitorum longus muscles were tested according to the Oxford scale for muscle strength ( 0 points for no muscle contraction, to 5 points for normal strength). To create a complete image of function, stability while standing on one leg, gait pattern, soft-tissue symptoms, sensitivity to touch and the localization and length of scars, were recorded.

Ultimately, the differences in clinical outcome after arthroscopic and open arthrolysis of the ankle joint, were compared.

The equivalence of distribution of age, sex, follow-up interval and initial KLS were evaluated in both the recruited and nonrecruited groups to determine whether the studied cohort results were representative of the whole population.

Statistical analysis. The statistical software IBM SPSS Statistics 24.0 (IBM Corp., Armonk, NY, USA) was used to analyse the data. Survival and progression-free-survival were demonstrated with Kaplan-Meier graphs. For comparing arthroscopic and open surgery,
Cox regression, t-test, Mann-Whitney $U$-test, Pearson chi-squared test and binomial distribution were used, depending on the parameter being analysed. ANOVA was utilised for comparing outcome parameters between group with different follow-up intervals. The impacts of risk factors were examined by means of $t$-test, Pearson chi-squared test and bivariate correlation depending on the parameter being analysed.

\section{Results}

Enrolment and cohort characteristics. All patients who underwent open or arthroscopic ankle arthrolysis at a university-affiliated level 1 trauma centre between 2000 and 2014 were retrospectively evaluated. Within this time, 137 cases of arthrolysis of the ankle joint were performed in 137 patients. Thirty-five out of these 137 patients (34\%) did not meet inclusion criteria, were lost to follow-up or had died in the interim period. One-hundred and two (38 females/64 males) of these $(74 \%)$ were recruited for this study. Twentyseven patients $(26 \%)$ agreed to an interview via telephone but declined clinical and radiological re-evaluation at followup. Another 24 patients (24\%) completed clinical questionnaires. A complete set of questionnaires, examinations and radiographs was achieved in 51 patients (50\%) (Figure 1) (Table I).

The mean age at surgery was 42.6 years (range 14 to 76 years). The cohort was divided into 2-year interval groups to examine the progression of osteoarthrosis. The mean follow-up was 66.9 months (range 4 to 186 months). BMI varied between 18.5 and $46.0 \mathrm{~kg} / \mathrm{m}^{2}$, with a mean of 29.0 $\mathrm{kg} / \mathrm{m}^{2}$. At the time of surgery, seven (10.4\%) patients suffered from diabetes and $23(33.8 \%)$ were smokers. Twenty $(29.4 \%)$ patients continued to smoke at their most recent follow-up. The osteoarthrosis was post-traumatic in 91 patients, six of these also suffered from rheumatoid arthritis. Eleven suffered from idiopathic osteoarthrosis, six of these also suffered from gout which was diagnosed in joints other than the ankle.

Patient outcome scores and questionnaires. The primary endpoint of ankle replacement or fusion was reached in 16 patients $(15.7 \%)$ at the time of follow-up. The survival probability was $0.920 \pm 0.027$ at a minimum of 24 -month follow-up, decreasing to $0.853 \pm 0.038$ at 72 -month follow-up and $0.755 \pm 0.068$ at 10 -year follow-up, and remained constant for the following 5 years (Figure 2).

The progress of osteoarthrosis in the ankle joint was analysed by comparing FFI, AOFAS, Hannover score, VAS, walking distance, KLS and ROM of the ankle joint between the 2-year interval groups. The mean FFI was 33 points (range=8-72) for pain and 45 points (range=10-90) for function. The mean AOFAS was 64 points (range=12-90) and the mean Hannover score 57 points (range=23-88). Twenty-seven $(37.5 \%)$ patients reported a pain-free walking 


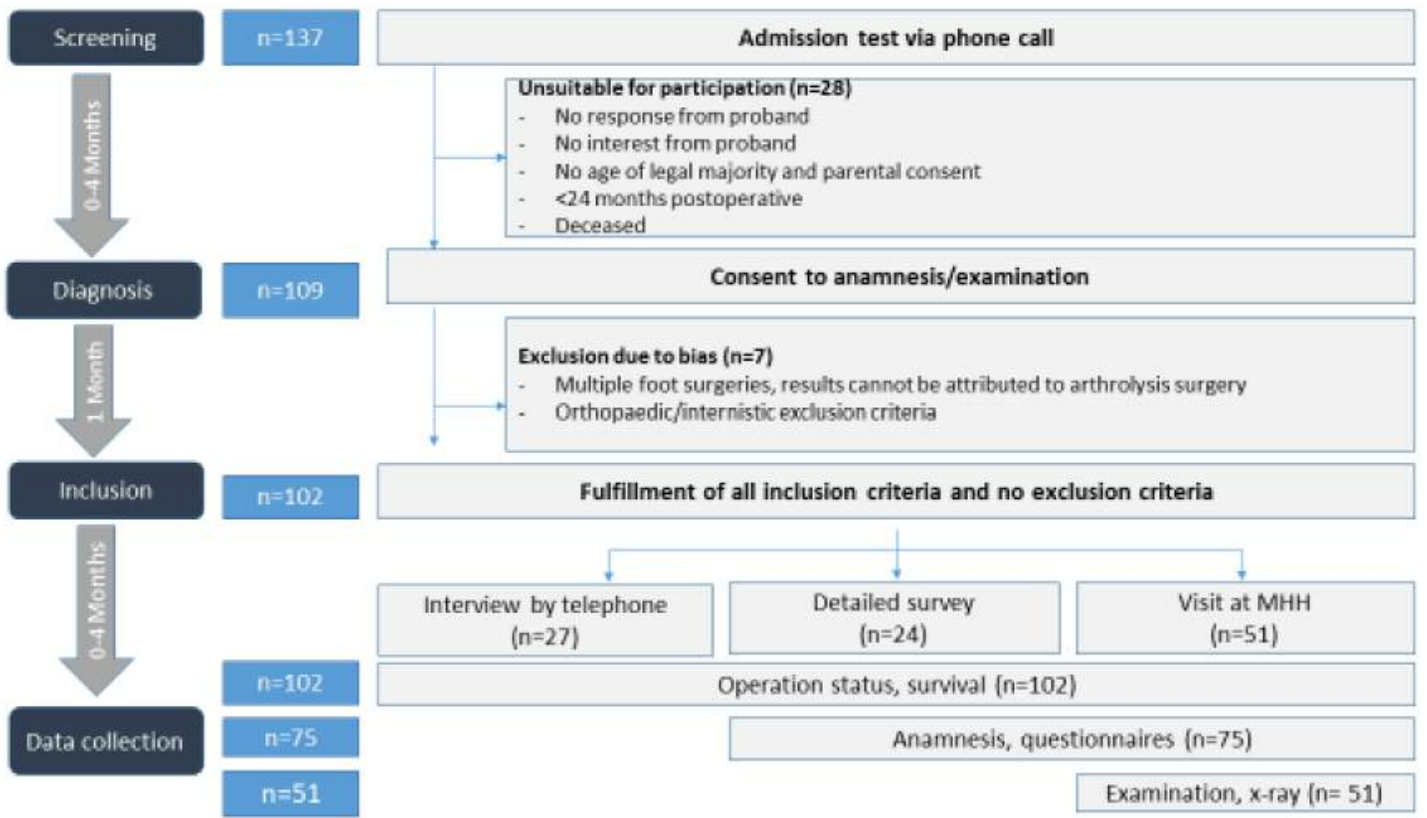

Figure 1. Study structure and recruitment for data acquisition.

distance of more than $2 \mathrm{~km}, 31(43 \%)$ reported between $500 \mathrm{~m}$ and $2 \mathrm{~km}$ and $14(19.4 \%)$ less than $500 \mathrm{~m}$. The mean VAS at follow-up was 3.6 (range=0-8), the mean KLS was 2.3 (range $=0-3$ ), and the mean ROM of the ankle joint was $39^{\circ}$ (range $=0-65$ ). None of the questionnaires, clinical or radiological findings revealed a significant difference between the 2-year-interval groups. A trend of improvement was suggested up to 4 years, no significant change from years 4-6, followed by improvement between 6-10 years and worsening symptoms from 10 years onwards (Figure 3).

The progression-free interval after surgery was detected by VAS. The probability of pain relief at a minimum of 12 months' follow-up was $0.55 \pm 0.06$, declining to $0.34 \pm 0.06$ at 72 months, and to $0.25 \pm 0.06$ at 96 months, which remained the same for the next 5 years.

Imaging. Examination of the weight-bearing pattern on the mirror-table showed a normal longitudinal arch in $76 \%$, a pes planus pattern in $14 \%$, and a pes cavus pattern in $10 \%$ of patients. A swollen hind foot appeared in $26 \%$ of all joints. Pes equinus was seen in $10 \%$ and splayfoot in $24 \%$ of the patients; $20 \%$ of the hindfoot axes showed valgus deviation of at least $10^{\circ}$, while $2 \%$ showed varus deviation.

The improvement of KLS between preoperative status and follow-up was two grades in one patient (2\%) and of one grade in 10 patients $(24 \%)$. No changes in the KLS were found in 18 patients (44\%). A worsening by one grade was found in 11 patients $(27 \%)$, and two grades in another patient $(2 \%)$.

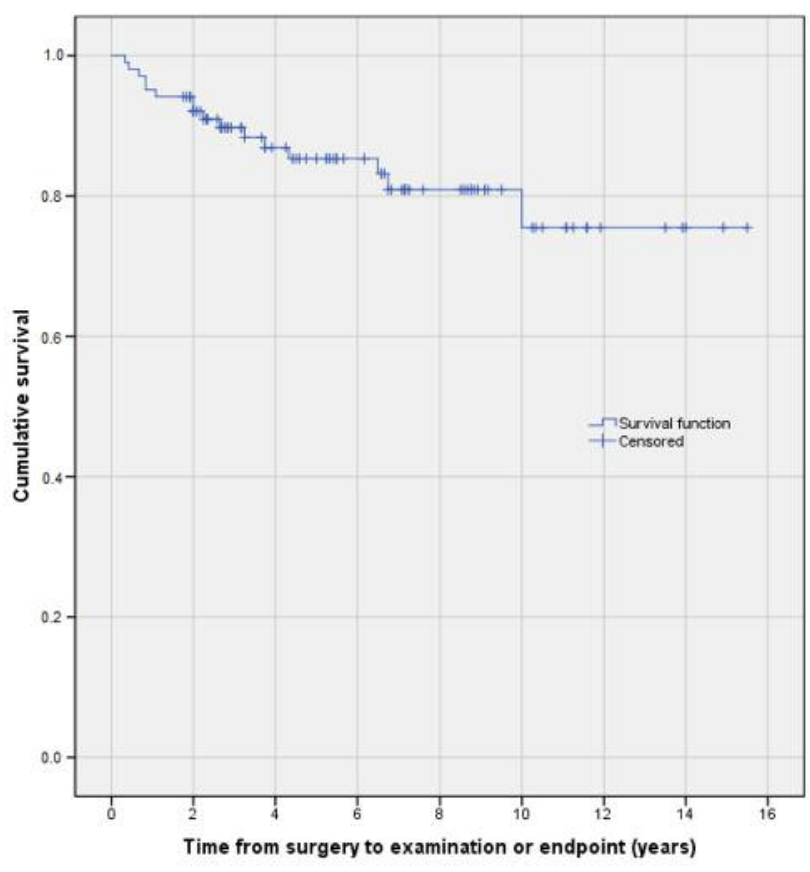

Figure 2. Kaplan-Meier estimator for ankle survival in 102 patients after arthrolysis surgery.

Related joints exhibited minor increases in osteoarthrosis. An increase of 0.7 (range=-3-3) points was found in the Chopart joint and a mean of 0.4 (range $=-3-3$ ) points in the subtalar joint. 

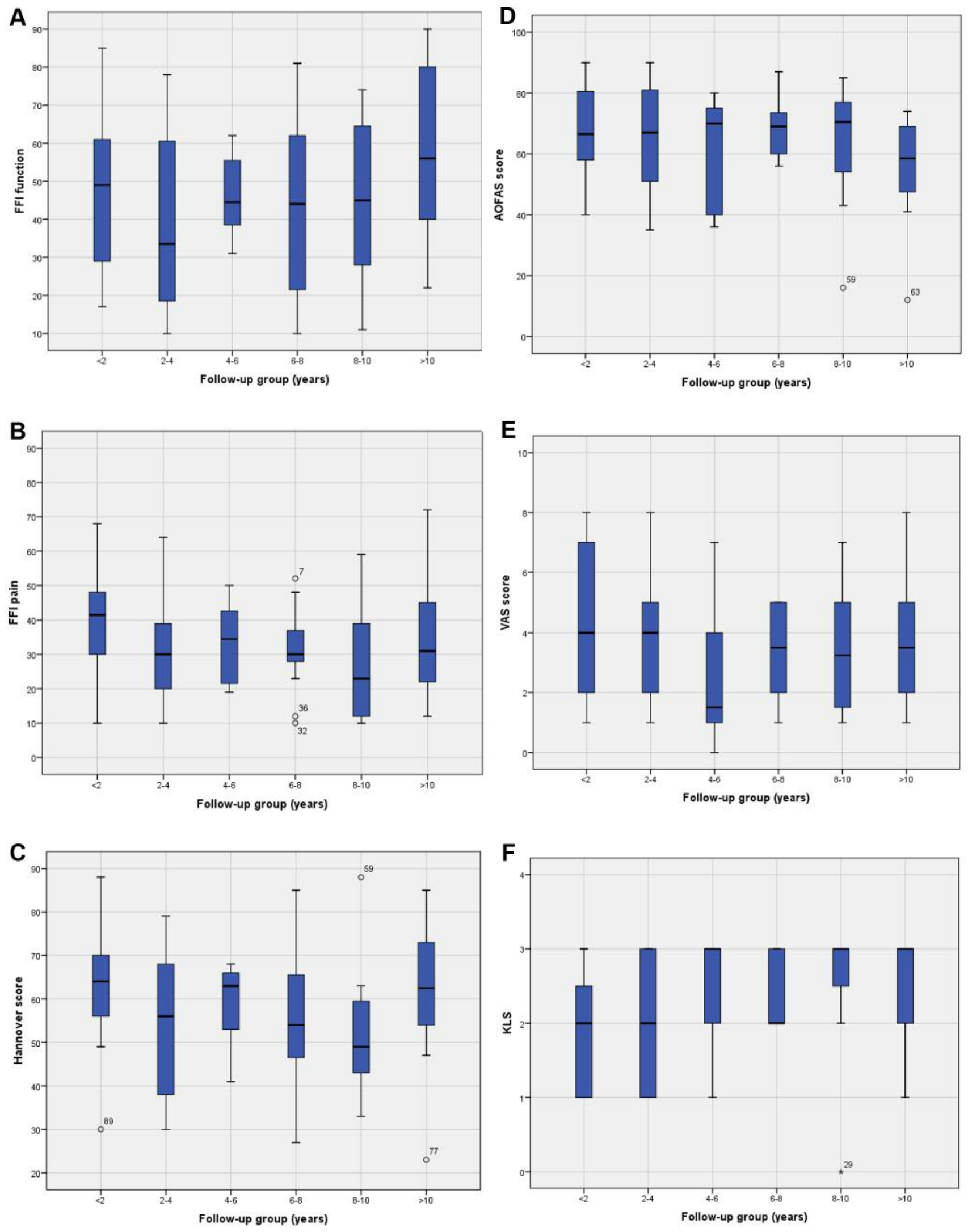

Figure 3. Continued 


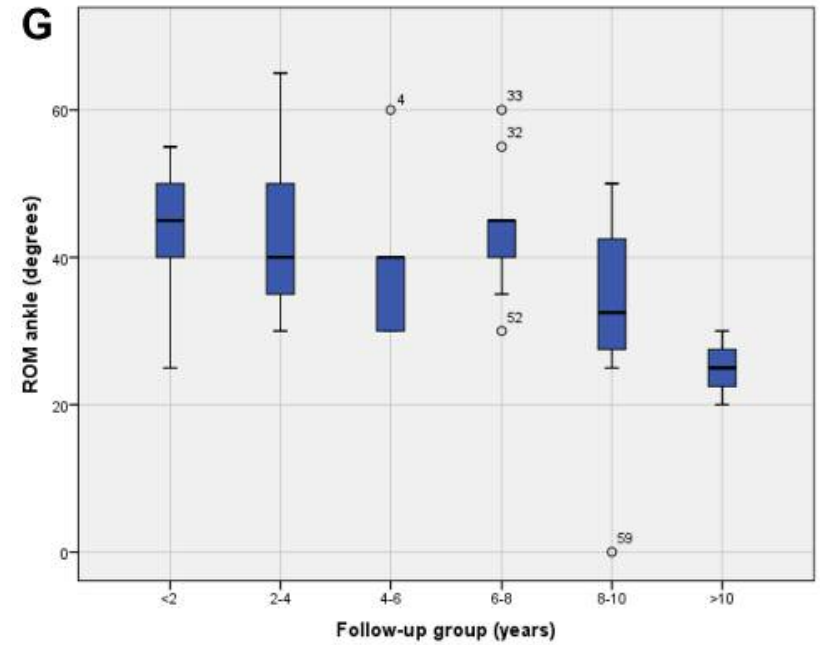

Figure 3. Comparison of selected outcomes between 2-year follow-up interval groups. A: Foot Function Index (FFI) for function; B: FFI for pain; C: Hannover score; D: American Orthopedic Foot and Ankle Score (AOFAS); E: Visual Analogue Scale (VAS); F: Kellgren-Lawrence Score (KLS); G: range of motion (ROM) for the ankle. The patients were divided into six groups according to their 2-year follow-up interval. The lines in the middle of boxes represent the median value. The lower half of the box includes the 25th to 50th percentile, the upper half of the box includes the 50th to 75th percentile. The whiskers above and below the boxes include $95 \%$ of all values. The outliers are defined as $5 \%$ of values outside the whiskers. Stars (for KLS) represent cases that have values more than three times the height of the box.

Functional examination. The mean ROM for extension and flexion of the treated ankle joint, with 90-degree flexion of the ipsilateral knee, was $39^{\circ}$ (range $\left.=0-65^{\circ}\right)$. The untreated side had a mean ROM of 56 (range $\left.=10-65^{\circ}\right)$. A limitation of more than $10^{\circ}$ degrees between treated and untreated degrees side was regarded as a detectable difference. $13 \%$ of patients exhibited this type of difference in ROM of hip extension and flexion, $14 \%$ in the hip rotation, $6 \%$ in the knee extension and flexion, $54 \%$ in the subtalar and $9 \%$ in the Chopart joints. A difference of $2 \mathrm{~cm}$ in the limb diameter (at the points described in Patients and Methods) was regarded as a detectible difference. Only $10 \%$ of all patients exhibited $2 \mathrm{~cm}$ or greater difference at one of the measured points.

A total of 37 patients $(74 \%)$ demonstrated full strength $(5 / 5)$ in all tested muscles. In cases of reduced strength, extensors $(24 \%)$ were more often affected than flexors $(16 \%)$.

Twenty-seven patients (53\%) suffered from soft-tissue complaints at follow-up, most commonly altered sensation around the incision.

For five patients $(10 \%)$, standing on one leg did not cause any problems; 28 patients $(55 \%)$ showed moderate instability while standing on one leg but for 18 patients (35\%), this was very difficult or not possible. As gait is a very subjective

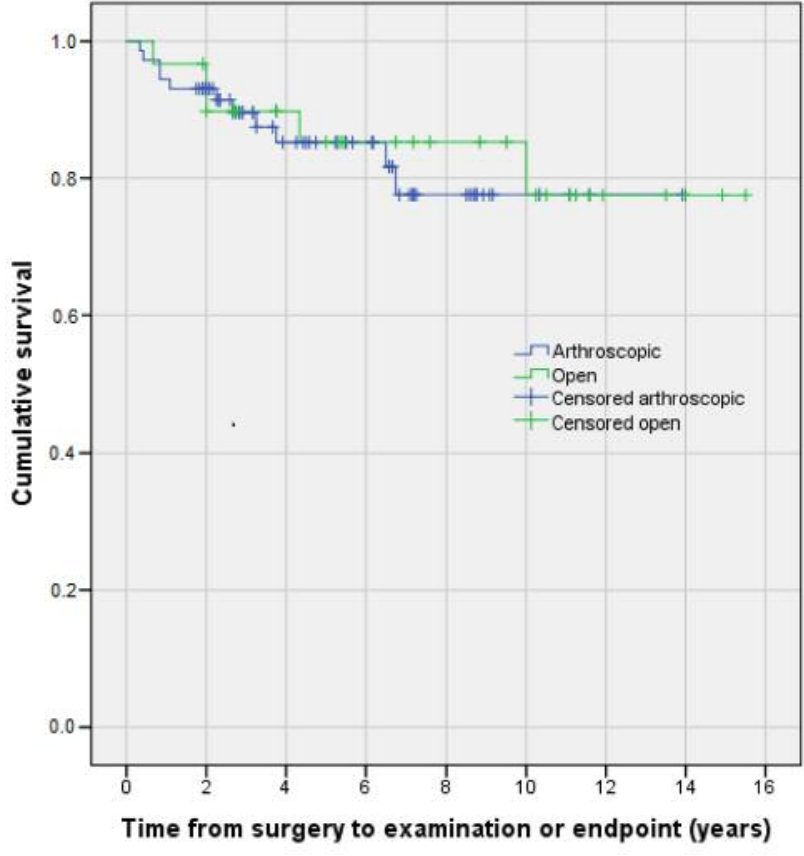

Figure 4. Kaplan-Meier graph of ankle survival in 102 patients postarthrolysis surgery according to treatment type.

parameter, two examiners (FRI and RG/UW) observed it independently concluding that 29 patients $(57 \%)$ demonstrated a normal gait, 16 (31\%) showed moderate limping when walking barefoot and six (12\%) showed an irregular gait whilst wearing shoes and had significant difficulties walking barefoot. Five patients required assistance with a cane and one required two crutches.

Comparison arthroscopic vs. open treatment. Comparing arthroscopic and open procedures, tests demonstrated no significant difference $(p=0.872)$ in respect of the survival of the joint (Figure 4). The progression-free survival $(p=0.867)$, FFI pain $(p=0.282)$, FFI function $(p=0.376)$ and improvement of patient satisfaction before and after surgery $(p=0.188)$ did not reveal any significant differences between the groups. Neither the range of motion nor the radiographic developments of ankle osteoarthrosis were different. The KLS of the Chopart joint increased significantly more after open (1.4 points) compared to arthroscopic ankle release (0.6 points) $(p=0.016)$. Patients treated with open ankle release suffered significantly more frequently from soft-tissue complaints such as altered skin sensation and pain $(83 \%$ vs. $44 \%)(p=0.015)$.

Personal impact factors for radiological and clinical outcomes. Sex was found to influence different parameters. The FFI function was significantly better in females 
Table I. Patient data.

\begin{tabular}{|c|c|c|c|c|}
\hline \multirow[b]{2}{*}{ Characteristic } & \multicolumn{4}{|c|}{ Study method } \\
\hline & $\begin{array}{l}\text { Telephone interview } \\
\qquad(\mathrm{n}=27)\end{array}$ & $\begin{array}{l}\text { Questionnaire } \\
\quad(\mathrm{n}=24)\end{array}$ & $\begin{array}{l}\text { Clinical and radiological } \\
\text { examination }(\mathrm{n}=51)\end{array}$ & $\begin{array}{c}\text { Total } \\
(\mathrm{n}=102)\end{array}$ \\
\hline Mean age at surgery, years & 39 & 46 & 42 & 43 \\
\hline Female gender, $\mathrm{n}(\%)$ & $8(29.6 \%)$ & $8(33.3 \%)$ & $22(43.1 \%)$ & 38 \\
\hline Ankle fusion, $\mathrm{n}(\%)$ & $4(14.8 \%)$ & $12(50 \%)$ & 0 (not invited) & 16 \\
\hline Mean survival (months) & 70 & 52 & 72 & 67 \\
\hline Traumatic cause, n (\%) & $26(96.3 \%)$ & $19(79.2 \%)$ & $46(90.2 \%)$ & $91(89.2 \%)$ \\
\hline Mean FFI pain & & 39.79 & 29.73 & 32.95 \\
\hline Mean FFI function & & 47.50 & 43.92 & 45.07 \\
\hline Mean Hannover score & & 59.83 & 55.63 & 56.98 \\
\hline Pain-free walking $>2 \mathrm{~km}, \mathrm{n}(\%)$ & & $5(23.8 \%)$ & $22(43.1 \%)$ & $27(37.5 \%)$ \\
\hline Mean VAS & & 4.4 & 3.2 & 3.6 \\
\hline Mean pre-operative KLS & & & 2.3 & 2.3 \\
\hline Mean post-operative KLS & & & 1.4 & 1.4 \\
\hline Mean KLS at follow-up & & & 2.3 & 2.3 \\
\hline Mean AOFAS & & & 64 & 64 \\
\hline Smoker, n (\%) & & & $20(29.4 \%)$ & $20(29.4 \%)$ \\
\hline Mean BMI, $\mathrm{kg} / \mathrm{m}^{2}$ & & & 29 & 29 \\
\hline Mean ankle ROM & & & $40^{\circ}$ & $40^{\circ}$ \\
\hline Perimeter difference $>2 \mathrm{~cm}, \mathrm{n}(\%)$ & & & $5(9.8 \%)$ & $5(9.8 \%)$ \\
\hline Soft-tissue complaints & & & $27(52.9 \%)$ & $27(52.9 \%)$ \\
\hline
\end{tabular}

FFI: Foot function index, VAS: Visual Analogue Scale, KLS: Kellgren-Lawrence score, AOFAS: American Orthopedic Foot and Ankle Score, BMI: body mass index, ROM: range of motion.

$(38.8 \pm 18.8$, range $=10-80)$ than males $(49.3 \pm 22.0$, range $=10$ 90) $(p=0.035)$. The FFI-pain was also significantly better in females $(27.0 \pm 12.1$, range $=10-58)$ than in males $(36.8 \pm 16.6$, range $=10-72)(p=0.007)$.

Age at surgery was significantly correlated with clinical outcome. FFI function (Pearson correlation 0.27, $p=0.02$ ), FFI pain (Pearson correlation $0.26, p=0.027$ ).

A high preoperative KLS was correlated with FFI function (Pearson correlation 0.26, $p=0.027$ ) and the AOFAS (Pearson correlation $-0.28, p=0.043$ ) and the ROM of the ankle joint (Pearson correlation $-0.37, p=0.008$ ).

No correlation was found between pack-years amongst smokers or BMI and FFI, AOFAS, Hannover score, VAS, survival, progression-free survival, KLS at follow-up or increase of KLS, ROM, perimeter or muscle strength.

\section{Discussion}

The aim of the current study was to study postoperative changes in function and pain following arthrolysis surgery of the ankle joint. The study endpoint was defined as revision surgery at the ankle joint, such as repeat arthrolysis, ankle arthroplasty, or arthrodesis. Special focus was placed on the correlation between pain, and radiological and clinical findings.

Ankle joint release reduces the risk for salvage procedures such as ankle fusion or arthroplasty as well as chronic pain and restriction of motion. More than $90 \%$ of patients received no surgical intervention within the 2 years following surgery. At 2-years follow-up, nearly half of all treated patients experienced steadily satisfactory or improving results. After a period of 10 years, $25 \%$ of all treated ankle joints remained without any deterioration but another $25 \%$ required ankle fusion.

In 2007, Hassouna et al. reported on the progression to salvage ankle surgery in $28 \%$ of patients (80 patients, 29 females and 51 males, mean age 41 years) within 5 years, in a similar cohort of patients to those of the current study (4). We found that patients who had not met the primary endpoint after 2 years had a very good long-term prognosis. In 2013, Choi et al. found a significant improvement in the AOFAS score (6) and Parma et al. described an AOFAS score of $78 \pm 14.8$ after 24 months and of 71 at a mean follow-up of 105 months (7). The current study revealed a slightly lower score of 64 after a mean of 67 months' followup. Others observed an improvement in the AOFAS for 2 years and the greatest improvements after 6 months (6). Survivorship analysis after arthroscopic ankle release by Parma et al. showed a high failure rate within the first 24 months, followed by a plateau (4). In contrast to these findings, the long-term results in our cohort did not decline steadily. The best results were seen in patients at between 2 and 4 years and between 6 and 10 years. The first peak may 
be a consequence of complete healing of the scars. Overuse and recurrence of typical osteoarthrotic symptoms may lead to an increase in pain and decrease in function after more than 4 years. Lifestyle modifications might have been a factor in late improvement after 6 or more years as most patients reported a change to joint-friendly physical exercise at a later stage. Overall, 62 out of $75(83 \%)$ patients showed excellent or good results, which lies in the range between $87 \%$ (16) and $77 \%$ (5) reported in the literature.

A significantly different range of motion between treated $\left(39^{\circ}\right)$ and untreated ankles $\left(56^{\circ}\right)$ was found amongst our patients $(p<0.001)$. The mean postoperative ROM in previous studies was $50^{\circ}(p<0.004)(17), 34 \pm 6^{\circ}(6)$ and $45^{\circ}$ (18), while we recorded a mean of $39^{\circ}$ at a mean of 67 months' follow-up. A study with 406 ankles with osteoarthrosis (radiologic ankle grade greater than KLS 2) without surgical treatment revealed a smaller mean ROM for dorsal extension and plantar flexion of $22^{\circ}$ (1).

A decreasing ROM in the ankle joint can be compensated for by the improvement of movement in the subtalar and Chopart joints. This leads to a slightly restricted gait even in fused ankle joints. The higher load and extension of related joints can lead to osteoarthrosis. Signs and symptoms in the current study cohort were very mild. Radiographs at follow-up showed a less than $10 \%$ increase in features of osteoarthrosis in the subtalar and Chopart joints compared to images immediately postoperatively. Gaedke et al. found a higher incidence of osteoarthrosis in the subtalar and Chopart joints after ankle fusion in a similar setting with a mean follow-up of 9.6 years; secondary osteoarthrosis appeared in 39 and nine out of 69 patients, respectively (19). This underlines the importance of preserved mobility in the ankle joint in order to reduce the risk for osteoarthrosis of the related joints.

The differences in the ROM of the hip and the knee and lower limb diameters were mild and mostly seen in patients above the age of 50 years. None of the patients complained about consequent limitations in their daily routine. Nevertheless, those with a restriction of over $10^{\circ}$ or a diameter difference over $2 \mathrm{~cm}$, compared to the contralateral side, were recommended for further assessment.

Extensors were slightly weaker than flexors, which may be caused by a relieving posture to counteract pain and impingement in extension. Overall, $44 \%$ of the patients treated arthroscopically still suffered from mild soft-tissue complaints. This is about half as many as amongst the openly treated group (83\%), but more than those described by Martin et al. (9\%) (11).

Those patients who felt unstable whilst standing on one leg often reported repeated ankle sprains prior to surgery. Insufficient integrity of the supporting lateral and medial ankle ligament complexes might explain this.

Patients who demonstrated difficulties when walking barefoot responded well to rocker-bottom soled shoes.
It became apparent that sex had significant effects on the study outcome. On average, women reported $10 \%$ less pain and better function $(p=0.007)$. Neither Choi et al. (5) $(p=1.22)$ nor Parma et al. $(p=0.96)$. found this correlation with gender at any follow-up point (7).

The patient's age had an effect on ankle joint extension and the radiographic appearance of the adjacent joints. Younger patients scored significantly better with regard to these parameters, making age a significant prognostic factor. The impact of advanced age and gender has been a subject of former studies. Age did not appear to be a significant prognostic factor in two studies $(4,6)$, whilst Hassouna et al. found that patients who had salvage ankle surgery were on average 11 years older, although no significant difference was found (4). Two further studies, with a comparable number of participants and with over 1,300 participants, respectively, ascertained higher age to be a negative outcome factor $(7,9)$. This effect can also be seen in the conservative treatment of osteoarthrosis (20).

The KLS has been recommended to assess the radiographic signs of osteoarthrosis of the ankle (15). Nevertheless, KLS is poorly predictive for long-term clinical outcome. KLS after surgery is similar in moderately and mildly affected joints. As surgery leads to similar radiological results, patients with mild to moderate osteoarthrosis are suitable for the procedure as noted previously $(21,22)$. Cerulli et al. did not find any correlation between radiological findings of osteoarthrosis and clinical outcome (16). In patients with progressive narrowing of the joint space, the reappearance of osteophytes happens more quickly (5). A total of $74 \%$ of our patients did not manifest any changes or only a minor increase in osteoarthrosis at follow-up.

The mean outcomes of patients with pes planus and pes cavus were not significantly different but a tendency towards worse clinical results in the presence of pes cavus $(p=0.17)$ was shown in our study.

Previously the BMI was considered to be a factor in poor outcomes as ascertained using the AOFAS and VAS scoring systems (6). Our study found only mild effects of BMI, which could be explained by medical comorbidities.

It is very likely that patients who suffer from idiopathic polyarthrosis or rheumatoid arthritis will suffer from osteoarthrosis in different joints in the future. Similarly to patients after ankle injury, they have high loads across adjacent joints in dorsal extension.

We found a traumatic cause of osteoarthrosis led to a significantly higher rate of joint stiffness than any other cause, which was in accordance with similar studies on posttrauma ankle osteoarthrosis (7) and intra-articular lesions (6) as prognostic parameters. The level of osteoarthrosis we recorded in posttraumatic patients supports findings by Regan et al. in which $63 \%$ of 281 patients demonstrated evidence of radiographic osteoarthrosis at follow-up, $31 \%$ were mild, $22 \%$ moderate, and $10 \%$ severe (23). 
The results of the current study are limited by the retrospective design, a combination of externally and internally measured parameters, and a heterogeneous cohort. Even though there is a certain commonality in terms of the steps in ankle release surgery, different surgeons and surgical techniques over a period of 14 years will have had an inevitable influence on the results. There is potential for recall bias in terms of the retrospectively ascertained VAS at time of surgery and before ankle fusion. Some patients had difficulties in remembering the exact pain level from several years prior to questioning and we had the impression that patients tended to let their current degree of satisfaction influence their memory of past symptoms.

\section{Conclusion}

Fewer than $20 \%$ of the current study cohort required ankle fusion or joint replacement after arthrolysis of the ankle joint. Open surgery had a negative effect on skin sensation, but not on function, pain or quality of life. Female gender and young age were found to be positive predictors for a good clinical outcome. Preoperative radiological findings and the interval after surgery are poorly correlated with progression of osteoarthrosis.

\section{Acknowledgements}

This study was financially supported by the KlinStrucMed programme of the independent Else Kröner Fresenius Foundation (Else-Kröner-Fresenius-Stiftung) for the clinical MD of FIR. The Authors also appreciate the educational support of the Hannover Biomedical Research School.

\section{References}

1 Valderrabano V, Horisberger M, Russell I, Dougall H and Hintermann B: Etiology of Ankle Osteoarthritis. Clin Orthop Relat Res 467(7): 1800-1806, 2009.

2 Saltzman CL, Salamon ML, Blanchard GM, Huff T, Hayes A, Buckwalter $\mathrm{J}$ and Amendola A: Epidemiology of ankle arthritis: report of a consecutive series of 639 patients from a tertiary orthopaedic center. Iowa Orthop J 25: 44-46, 2005.

3 Hassouna H, Kumar S and Bendall S: Arthroscopic ankle debridement: 5-year survival analysis. Acta Orthop Belg 73(6): 737-740, 2007.

4 Tol JL, Verheyen CPPM and Dijk CN Van: Arthroscopic treatment of anterior. J Bone Joint Surg Br 83: 9-13, 2001.

5 Choi WJ, Choi GW, Kwon $\mathrm{H}$ and Lee JW: Arthroscopic treatment in mild to moderate osteoarthritis of the ankle. Knee Surg Sport Traumatol Arthrosc 21(6): 1338-1344, 2013.

6 Parma A, Buda R, Vannini F, Ruffilli A, Cavallo M, Ferruzzi A and Giannini S: Arthroscopic treatment of ankle anterior bony impingement: The long-term clinical outcome. Foot Ankle Int 35(2): 148-155, 2014.

7 Deng DF, Hamilton GA, Lee M, Rush S, Ford LA and Patel S: Complications associated with foot and ankle arthroscopy. J Foot Ankle Surg 51(3): 281-284, 2012.
8 Zengerink M and van Dijk CN: Complications in ankle arthroscopy. Knee Surg Sport Traumatol Arthrosc 20(8): 1420-1431, 2012.

9 Epstein DM, Black BS and Sherman SL: Anterior ankle arthroscopy: indications, pitfalls, and complications. Foot Ankle Clin 20(1): 41-57, 2015.

10 Martin DF, Baker CL, Curl WW, Andrews JR, Robie DB and Haas AF: Operative ankle arthroscopy. Long-term follow-up. Am J Sports Med 17(1): 16-23, 1989.

11 Glazebrook MA, Ganapathy V, Bridge MA, Stone JW and Allard J-P: Evidence-based indications for ankle arthroscopy. Arthroscopy 25(12): 1478-1490, 2009.

12 Kostuj T, Krummenauer F, Schaper K, Stief F, Zettersten K, Baums MH, Meurer A and Lieske S: Analysis of agreement between the German translation of the American Foot and Ankle Society's Ankle and Hindfoot Scale (AOFAS-AHS) and the Foot Function Index in its validated German translation by Naal et al. (FFI-D). Arch Orthop Trauma Surg 134(9): 1205-1210, 2014.

13 Thermann H, Hüfner T, Schratt H-E, Held C and Tscherne H: Subtalar fusion after conservative or operative treatment of intraarticular calcaneus fracture. Unfallchirurg 102(1): 13-22, 1999.

14 Kraus VB, Kilfoil TM, Hash TW, McDaniel G, Renner JB, Carrino JA and Adams S: Atlas of radiographic features of osteoarthritis of the ankle and hindfoot. Osteoarthr Cartil 23(12): 2059-2085, 2015.

15 Holzer N, Salvo D, Marijnissen ACA, Vincken KL, Ahmad AC, Serra E, Hoffmeyer P, Stern R, Lübbeke A and Assal M: Radiographic evaluation of posttraumatic osteoarthritis of the ankle: the Kellgren-Lawrence scale is reliable and correlates with clinical symptoms. Osteoarthr Cartil 23(3): 363-369, 2015.

16 Cerulli G, Caraffa A, Buompadre V and Bensi G: Operative arthroscopy of the ankle. Arthrosc J Arthrosc Relat Surg 8(4): 537-540, 1992.

17 Bauer T, Breda R and Hardy P: Anterior ankle bony impingement with joint motion loss: The arthroscopic resection option. Orthop Traumatol Surg Res 96(4): 462-468, 2010.

18 Arnold H: Posttraumatic impingement syndrome of the ankleIndication and results of arthroscopic therapy. Foot Ankle Surg 17(2): 85-88, 2011.

19 Gaedke IE, Wiebking U, O’Loughlin P, Krettek C and Gaulke $\mathrm{R}$ : Clinical and radiological mid- to long-term outcomes folowing ankle fusion. In Vivo 32: 1463-1471, 2018.

20 Thomas AC, Hubbard-Turner T, Wikstrom EA and PalmieriSmith RM: Epidemiology of Posttraumatic Osteoarthritis. J Athl Train 52(6): 491-496, 2017.

21 Castagnini F, Pellegrini C, Perazzo L, Vannini F and Buda R: Joint sparing treatments in early ankle osteoarthritis: current procedures and future perspectives. J Exp Orthop 3(1): 3, 2016.

22 Weatherall JM, Mroczek K, McLaurin T, Ding B and Tejwani N: Post-traumatic ankle arthritis. Bull Hosp Jt Dis 71(1): 104112, 2013.

23 Regan DK, Gould S, Manoli A and Egol KA: Outcomes over a decade after surgery for unstable ankle fracture. J Orthop Trauma 30(7): e236-241, 2016. 